\title{
Tunable Terahertz Wavelength Conversion Based on Optofluidic infiltrated Rib Silicon Waveguides
}

\section{H. Pakarzadeh}

Shiraz University

S. Hosseinabadi

Shiraz University

Iraj Sadegh Amiri ( $\square$ irajsadeghamiri@tdtu.edu.vn )

TDTU https://orcid.org/0000-0001-8121-012X

\section{Research Article}

Keywords: Silicon waveguide, photonic crystal, optical fluids, four-wave mixing, dispersion engineering, terahertz

Posted Date: April 2nd, 2021

DOI: https://doi.org/10.21203/rs.3.rs-363782/v1

License: (c) (i) This work is licensed under a Creative Commons Attribution 4.0 International License. Read Full License 


\title{
Tunable Terahertz Wavelength Conversion Based on Optofluidic Infiltrated Rib Silicon Waveguides
}

\author{
H. Pakarzadeh ${ }^{1}$, S. Hosseinabadi ${ }^{1}$, and I. S. Amiri ${ }^{2,3}$ \\ ${ }^{1}$ Physics Department, Shiraz University of Technology, Shiraz, Iran \\ ${ }^{2}$ Computational Optics Research Group, Advanced Institute of Materials Science, \\ Ton Duc Thang University, Ho Chi Minh City, Vietnam \\ ${ }^{3}$ Faculty of Applied Sciences, Ton Duc Thang University, Ho Chi Minh City, Vietnam \\ E-mail: pakarzadeh@sutech.ac.ir \\ E-mail: sh hosseinabadi@yahoo.com \\ E-mail: irajsadeghamiri@tdtu.edu.vn*
}

\begin{abstract}
Terahertz (THz) sources for various applications have attracted especial attention. Compared with the standard silica-based optical fibers, silicon waveguides have advantages such as higher refractive index and lower absorption loss over the $\mathrm{THz}$ region. In this paper, for the first time, a rib silicon waveguide based on the photonic crystal (PC) idea is designed and by using the scalar modulation instability (SMI) phenomenon, tunable wavelength conversion for generation of $\mathrm{THz}$ radiation is simulated. By changing the structural parameters such as the air-hole diameter of the PC and infiltration of optical fluids into the air holes, linear and nonlinear characteristics of the waveguide are controlled and hence the generated $\mathrm{THz}$ radiation is considered as a tunable source. Simulation presents the maximum converted wavelength of $326.17 \mu \mathrm{m}$ is obtained when the air-hole diameter is set at $\mathrm{d}=0.86 \mu \mathrm{m}$, where pump wavelength is in the normal dispersion regime. Also, we have infiltrated optical fluids into PC air holes in order to change the dispersion properties of the waveguide while keeping the geometrical parameters unchanged. This led to converted wavelengths of 70.5, 76.8 and $107.5 \mu \mathrm{m}$, all located in the terahertz region. The loss of this waveguide is less than $0.8 \mathrm{~dB} / \mathrm{cm}$, which is less than that of previously reported in similar silicon waveguides.
\end{abstract}

Keywords: Silicon waveguide; photonic crystal; optical fluids; four-wave mixing; dispersion engineering; terahertz.

\section{Introduction}

Terahertz $(\mathrm{THz})$ radiation as an electromagnetic radiation in the frequency range of 0.1 to $10 \mathrm{THz}$ (wavelength range of $30 \mu \mathrm{m}$ to $3 \mathrm{~mm}$ ) has attracted a lot of interest in both science and technology [1]. The THz band which is located between the infrared and microwave region is a low energy and nonionized radiation that may penetrate in non-polar and non-metallic materials such as cloth, paper, stone and plastics [2].

The development of efficient terahertz wave sources is important for measurement and biology [3]. The terahertz radiation is generated by various ways such as photoconductive antennas [4], large particle accelerators [5, 6], quantum cascade lasers [7], and optical rectification in nonlinear crystals [8, 9]. The important ones that have been recently used is four-wave mixing (FWM) [10] in silicon waveguides. 
Compared to the conventional silica-based optical fibers, a rib silicon waveguide is a more consistent structure for generating THz waves through the FWM. Inherent advantages of the silicon waveguide over the silica fiber include the absorption loss of the silicon which is less than $0.23 \mathrm{~cm}^{-1}$ in $1.2-6.9$ micron and 25-200 micron [11], while in optical fiber absorption coefficient is in the terahertz region which is $5 \mathrm{~cm}^{-}$ 1; silicon nonlinear refractive index is 200 times greater than the silica [12]; the refractive index (about 3.5) of the silicon is much larger than the air, which represents a very strong light confinement; the rib silicon waveguide is compatible, where it enables large-scale integration with a low cost [10].

Owing to the interplay of nonlinear effects and dispersion, many nonlinear systems can exhibit an unstable phenomenon known as the modulation instability (MI) which results in a stable mode modulation [13]. MI is a third-order nonlinear phenomenon that can be utilized in optical fibers/waveguides for wavelength conversion [13]. The simplest form of MI is considered as scalar modulation instability (SMI) where there is no dependency on the polarization state of the lightwave [13]. The underlying physics of SMI is the production of new photons: known as Stokes and anti-Stokes. In the case of SMI, for satisfying the phase-matching condition, $\lambda_{\mathrm{p}}$ must be adjusted near the ZDW which stands for the zero-dispersion wavelength as $\lambda_{0}$ of the waveguide for $\lambda_{p}<\lambda_{0}$ or $\lambda_{p}>\lambda_{0}$ dispersion regimes.

In this paper, we design for the first time a photonic crystal-based rib silicon waveguide for tunable wavelength conversion in the terahertz region. We examine wavelength conversion via the SMI phenomenon in both the anomalous and the normal dispersion regimes. To this end, we change the structural parameters (both geometry and optofluidic infiltration) of the waveguide to engineer the dispersion and nonlinear characteristics and hence to control the phase-matching condition and finally tune the converted wavelengths in $\mathrm{THz}$ region. As the wavelength conversion strongly depends on groupvelocity dispersion (GVD) parameter $\beta_{2}$, the merit of the optofluidic infiltration is to obtain tunable wavelength conversion while keeping the waveguide geometry unchanged.

The optical properties of fluids are generally very wide. An optical fluid can be applied to any photonic device where there is an air hole $[14,15]$. The fluid interacts with the confined field and modifies the properties [15]. Fluid can be successfully infiltrated in photonic crystal (PC) and microstructures and change the waveguide properties. Most recently, the optofluidic infiltration in photonic crystal fibers (PCFs) has been used for wavelength conversion in the telecommunication region [16]. Therefore, in this work, we intend to investigate the wavelength conversion in the PC-based rib silicon waveguide to access the $\mathrm{THz}$ region not only with the help of geometrical changes but also with optofluidic infiltration possessing various refractive indices. In fact, by the optofluidic infiltration into the PC air holes of the waveguide and by changing the optical fluid's refractive index $n_{F}$, the dispersion properties can be changed and thereby the converted wavelengths are tuned [17].

The paper structure is as below. In Section 2, the theoretical background of the wavelength conversion in both the anomalous and the normal dispersion regimes based on the SMI is presented. In Section 3, we design a PC-based rib silicon waveguide and its linear and nonlinear characteristics are determined. In Section 4, the wavelength conversion is simulated when geometrical parameters are changed or the waveguide is infiltrated with various optical fluids. Finally, the paper is concluded in Section 5.

\section{Theory of the study}

MI is a general feature of the lightwave propagating in an optical medium where dispersion and nonlinear effects are simultaneously present. Based on MI, when a pump wave with the angular frequency of $\omega_{p}$ is input into the optical waveguide, the frequency of the sidebands (Stokes and Anti Stokes) are symmetrically located than the pump frequency (zero detuning); such that, the energy conservation 
condition is met, i.e. $2 \omega_{\mathrm{p}}=\omega_{\mathrm{a}}+\omega_{\mathrm{s}}$. The angular frequencies of the sidebands (Stokes and Anti Stokes) are defined respectively by $\omega_{\mathrm{s}}$ and $\omega_{\mathrm{a}}[16]$.

The frequency detuning of sidebands with respect to the pump frequency is given by $\Omega=\omega_{p}-\omega_{s}=$ $\omega_{a}-\omega_{p}$ where $\omega_{s}=\frac{2 \pi c}{\lambda_{s}}$ and $\omega_{a}=\frac{2 \pi c}{\lambda_{a}}$, where the Stokes and anti -Stokes wavelengths are presented by $\lambda_{s}$ and $\lambda_{a}[13,16]$.

Linear stability analysis, phase-matching condition, and FWM can be used to describe the SMI in an optical medium [13]. From the phase-matching condition, one can readily determine the peak frequency detunings $\Omega_{\text {max }}$, where the SMI maxima occurs, thus $\Delta k=\beta_{2} \Omega^{2}+2 \gamma p_{0}=0$. Therefore, the position of peak frequency detunings in the anomalous dispersion regime are easily determined as $[13,16]$ :

$$
\Omega_{\max }= \pm \sqrt{\frac{2 \gamma p_{0}}{\left|\beta_{2}\right|}}
$$

where $p_{0}$ is the peak power of the input pump and $\beta_{2}$ is the second-order dispersion coefficient of the waveguide calculated at pump wavelength $\lambda_{p}$. Also, $\gamma$ denotes the nonlinear parameter of the waveguide. On the other hand, for the normal dispersion regime, the mode propagation constant $\beta$ should be expanded up to $\beta_{4}$ to determine $\Omega_{\max }$ as $[13,16]$ :

$$
\Omega_{\max }= \pm \frac{\sqrt{-2 \beta_{4}\left(3 \beta_{2}+\sqrt{9 \beta_{2}^{2}-6 \beta_{4} \gamma P_{0}}\right)}}{\beta_{4}}
$$

where the phase-matching criterion: $\Delta k=\beta_{2} \Omega^{2}+\frac{1}{12} \beta_{4} \Omega^{4}+2 \gamma p_{0}=0$ is used. It should be noted that in the anomalous dispersion regime where $\beta_{2}$ is negative, the first term of $\Delta k=\beta_{2} \Omega^{2}+2 \gamma p_{0}$ can be cancelled out by the second term (which is always positive) and fulfill the phase-matching condition. In the normal dispersion regime however, since $\beta_{2}$ is positive, the negative value is satisfied by $\beta_{4}$ so that the phase-matching condition $\Delta k=\beta_{2} \Omega^{2}+\frac{1}{12} \beta_{4} \Omega^{4}+2 \gamma p_{0}=0$ is fulfilled.

The intensity gain coefficient of the SMI in the anomalous dispersion regime can be calculated as $[13,16]$ :

$$
g(\Omega)=2 \operatorname{Im}\left[\sqrt{\frac{1}{2} \beta_{2} \Omega^{2}\left(\frac{\beta_{2} \Omega^{2}}{2}+2 \gamma p_{0}\right)}\right]
$$

Both theoretical and experimental show the SMI take places in the normal dispersion regime as well, thus the higher-order dispersion coefficients cannot be ignored. Therefore, considering dispersion terms up to the fourth-order coefficient $\beta_{4}$, the intensity gain coefficient in the normal dispersion regime becomes $[13,16]$ :

$$
g(\Omega)=\frac{1}{12} \operatorname{Im}\left[\left(\sqrt{\beta_{4} \Omega^{4}+12 \beta_{2}} \times \sqrt{\beta_{4} \Omega^{4}+12 \beta_{2} \Omega^{2}+48 \gamma p_{0}}\right) \Omega\right]
$$

The converted wavelength can reach the THz region.

\section{Designing the PC-Based Rib Silicon Waveguide}

In this section, we design a PC-based rib silicon waveguide with suitable dispersion characteristics and investigate the wavelength conversion in this waveguide for a given input pump wave. If the diameter of circular air holes of PC changes and hence tailoring the waveguide dispersion characteristics, the wavelength range is tuned. The waveguide that is studied in this work is made of silicon where its wavelength-dependent refractive index is given as [18]: 


$$
n^{2}-1=\frac{10.6684293 \lambda^{2}}{\lambda^{2}-(0.301516485)^{2}}+\frac{0.0030434748 \lambda^{2}}{\lambda^{2}-(1.13475115)^{2}}+\frac{1.54133408 \lambda^{2}}{\lambda^{2}-(1104)^{2}}
$$

The guidance mechanism of the waveguide is the same as the standard optical fibers, that is the total internal reflection [19].

Our proposal of the PC-based rib silicon waveguide is shown in Fig. 1 (a). The PC is arranged as a rectangular array of circular air holes inside the silicon waveguide background and is located at a distance of 14 microns from the top of the waveguide. The lattice pitch of the PC is fixed at $\Lambda=4.80 \mu \mathrm{m}$ and the wavelength conversion process is controlled by changing the diameter of the air holes. In Fig. 1 (b), the mode is well confined within the waveguide core located above the PC air holes. The air-hole diameter $\mathrm{d}$ can be changed for geometrical change of waveguide and then changing the waveguide dispersion. We have simulated the fundamental mode field distribution and mode propagation constant of the proposed waveguide [20-21]. A full-vectorial modal analysis based on the finite-difference eigenmode is used to obtain the field distribution of the fundamental mode and propagation constant of the waveguide [22, 23]. The method discretizes the waveguide cross section using a 100 numbers of mesh cells and mesh step of $1 \times 10^{-06} \mu \mathrm{m}$ in each direction and formulates Maxwell's equations in terms of a matrix eigenvalue problem. Perfectly matched layers with $18 \mu \mathrm{m}$ width are used as absorbing boundaries placed on the boundary of the waveguide. Conformal meshing was also applied to all material interfaces to reduce meshing errors and increase the numerical accuracy of the simulations.

It should be noted that our proposal is inspired by a silicon membrane waveguide reported by Z. Wang et. al. to generate terahertz radiation which made it possible to create new wavelengths only by changing its geometry [10]. However, our proposed PC-based rib silicon waveguide has more advantages than the silicon membrane waveguide or other designs [11] with unique features such as freedom in design owing to the PC air holes and much lower loss (less than $0.8 \mathrm{~dB} / \mathrm{cm}$,) in operating wavelengths. In addition, our waveguide enables generating new wavelengths without changing the geometry when using optofluidic infiltration in PC air holes. This makes our waveguide more suitable for industrial and commercial purposes in comparison with previous ones.

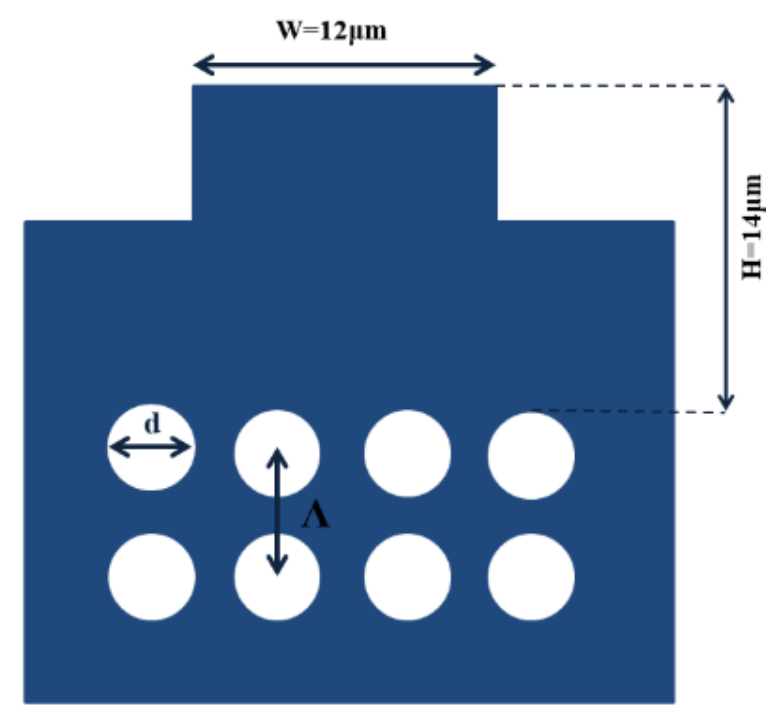

(a) 


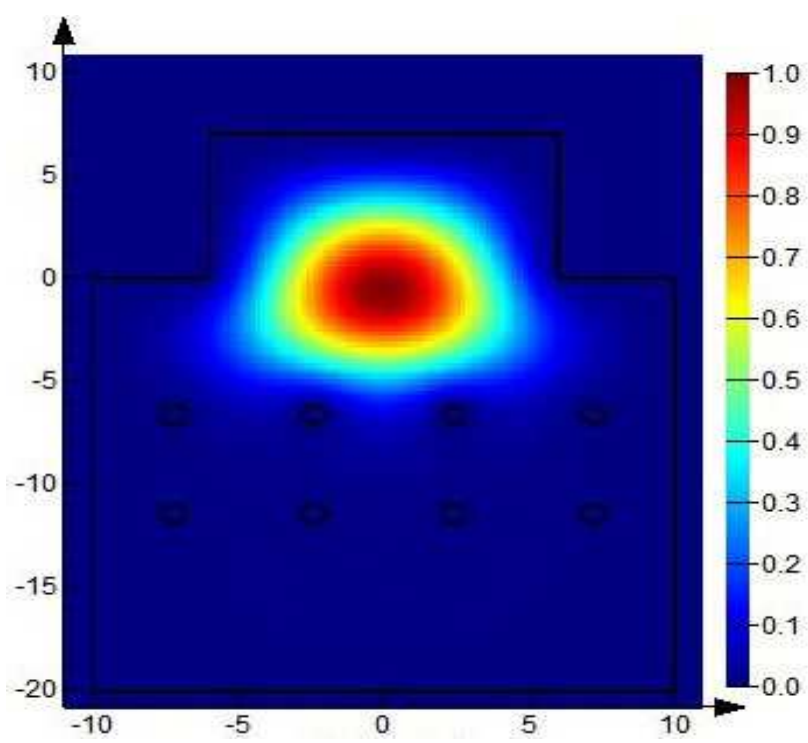

(b)

Fig 1. (a) Cross-sectional view of the PC-based rib silicon waveguide; (b) distribution of the fundamental TM mode confined in the waveguide core. The air-hole diameter $\mathrm{d}$ can be changed for geometrical change of waveguide.

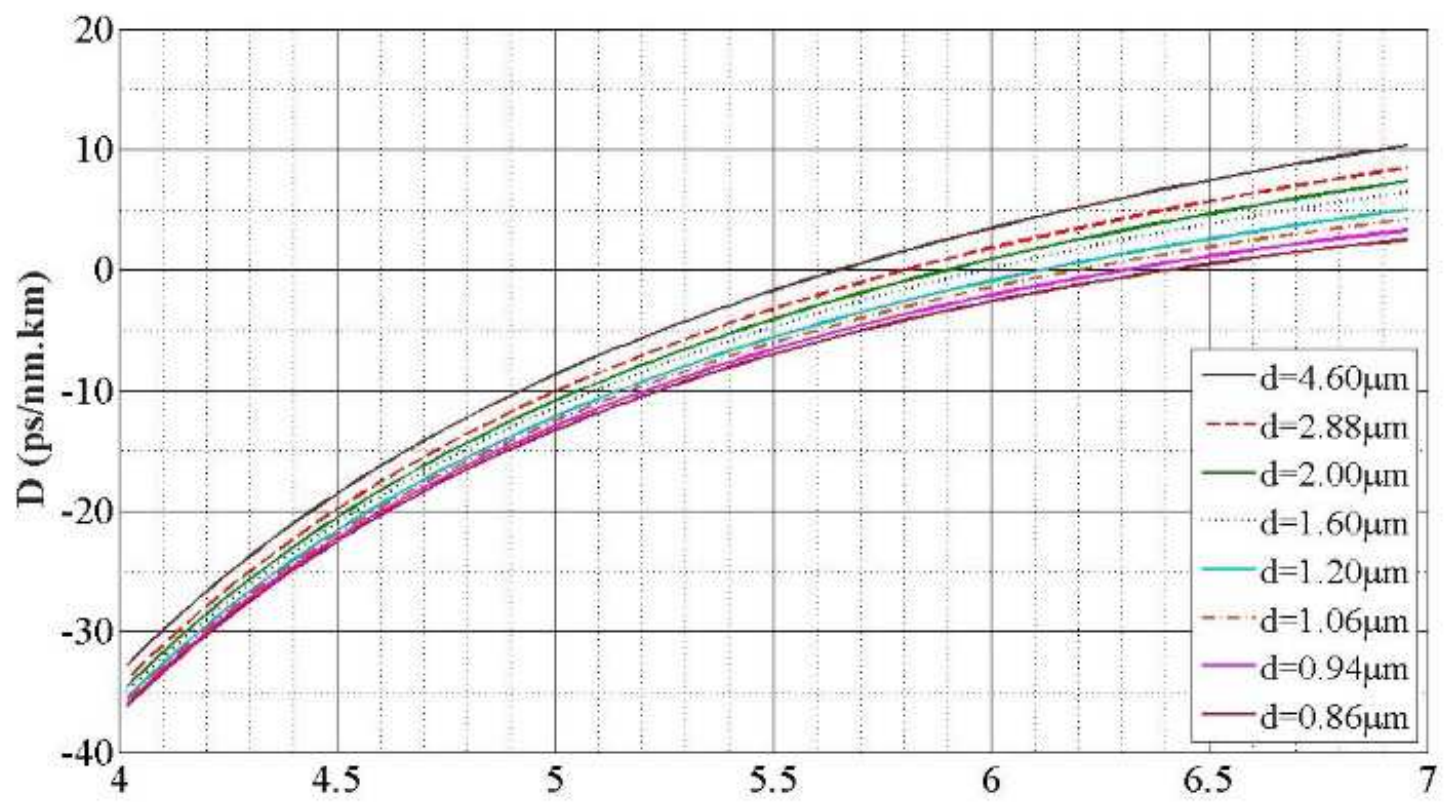

Fig 2. Dispersion curves D versus wavelength for different air-hole diameters d.

Fig. 2 shows dispersion curves $\mathrm{D}$ as a function of wavelength in the range of 4.0 to 7.0 microns for various air-hole diameters, d. As it is evident, by decreasing the diameter of the air holes, the zerodispersion wavelength (ZDW) shifts toward longer wavelengths; therefore, by engineering the dispersion curves, it is possible to tune the frequency shift and the gain of the converted wavelengths (see Eqs. (1)(4)). The dependency of the ZDW on the air-hole diameter is given in Table (1). 
Table 1. The zero-dispersion wavelength (ZDW) of the PC-based rib silicon waveguide for various air-hole diameters.

\begin{tabular}{|l|c|c|c|c|c|c|c|c|}
\hline $\mathrm{d}(\mu \mathrm{m})$ & 4.60 & 2.88 & 2.00 & 1.60 & 1.20 & 1.06 & 0.94 & 0.86 \\
\hline $\mathrm{ZDW}(\mu \mathrm{m})$ & 5.65 & 5.80 & 5.90 & 6.00 & 6.10 & 6.20 & 6.30 & 6.40 \\
\hline
\end{tabular}

\section{Tunable Wavelength Conversion in the PC-Based Rib Silicon Waveguide}

We simulate the tunable wavelength conversion by changing the waveguide geometry as well as by optofluidic infiltration in both normal and anomalous dispersion regimes. Then, the best situation for obtaining the wavelength conversion in the $\mathrm{THz}$ region is determined.

\section{4-1Tunable Wavelength Conversion via Geometrical Change in Anomalous Dispersion Regime}

As already mentioned, in the anomalous dispersion regime $\beta_{2}$ becomes negative. Using the dispersion curves D shown in Fig. 2 and Table (1), if the pump wavelength $\lambda_{\mathrm{p}}$ set at $6.60 \mu \mathrm{m}$, the second-order dispersion coefficient $\beta_{2}=-\frac{\lambda^{2}}{2 \pi c} \mathrm{D}$, becomes negative. By taking the second derivative of the $\beta_{2}$ with respect to the angular frequency, the fourth-order coefficient $\beta_{4}$ can be obtained which is reported in ref. [24].

The GVD parameter $\beta_{2}$, effective mode area $A_{\text {eff }}$ (or more intuitively the area of the intense spot seen in Fig. 1 (b)) and nonlinear parameter $\gamma$ of the PC-based rib silicon waveguide are listed in Table (2) for various air-hole diameters calculated at $6.60 \mu \mathrm{m}$ (this is because at this wavelength, for all air-hole diameters according to Table (1), the anomalous dispersion regime is fulfilled, i.e., $\lambda_{\mathrm{p}}>$ ZDW), and $\gamma=$ $\frac{n_{2} \omega_{p}}{c A_{\text {eff }}}=\frac{n_{2}}{\lambda_{p}} \frac{2 \pi}{A_{\text {eff }}}$, where $n_{2}=5 \times 10^{-18} \mathrm{~m}^{2} / W[10]$. Also, $n_{2}$ is the nonlinear refractive index of silicon.

Table 2. Linear as well as nonlinear parameters of the PC-based rib silicon waveguide for different diameters of air holes calculated at the pump wavelength of $\lambda_{P}=6.60 \mu \mathrm{m}$ in the anomalous dispersion region.

\begin{tabular}{|c|c|c|c|c|}
\hline $\mathrm{d}(\mu \mathrm{m})$ & $\begin{array}{c}\mathrm{D} \\
\left({ }^{2} / \mathrm{nm} . \mathrm{km}\right)\end{array}$ & $\beta_{2}\left({ }^{\left.p s^{2} / m\right)}\right.$ & $\begin{array}{c}A_{\text {eff }} \\
\left(\mu m^{2}\right)\end{array}$ & $\gamma(1 / W . m)$ \\
\hline 4.60 & 8.15 & $-1.89 \times 10^{-1}$ & 69.74 & $6.82 \times 10^{-2}$ \\
\hline 2.88 & 6.38 & $-1.48 \times 10^{-1}$ & 78.80 & $6.04 \times 10^{-2}$ \\
\hline 2.00 & 5.32 & $-1.23 \times 10^{-1}$ & 84.99 & $5.60 \times 10^{-2}$ \\
\hline 1.60 & 4.48 & $-1.04 \times 10^{-1}$ & 88.70 & $5.36 \times 10^{-2}$ \\
\hline 1.20 & 3.18 & $-7.36 \times 10^{-2}$ & 93.93 & $5.06 \times 10^{-2}$ \\
\hline 1.06 & 2.49 & $-5.77 \times 10^{-2}$ & 96.84 & $4.91 \times 10^{-2}$ \\
\hline 0.94 & 1.67 & $-3.87 \times 10^{-2}$ & 100.16 & $4.75 \times 10^{-2}$ \\
\hline
\end{tabular}




\begin{tabular}{|l|l|l|l|l|}
\hline 0.86 & 1.00 & $-2.32 \times 10^{-2}$ & 103.03 & $4.62 \times 10^{-2}$ \\
\hline
\end{tabular}

Also, by decreasing the air-hole diameters of the PC, the core-cladding index contrast is decreased and so $\gamma$ is decreased. Core-cladding index contrast means the difference between the refractive index of the core and that of the cladding. When the air-hole diameter of the PC in the cladding is decreased, the corecladding index contrast is decreased as well. This is because the core purely consists of $\mathrm{Si}$ with the refractive index of $\sim 3.5$ while the cladding consists of $\mathrm{Si}$ and/or air. When the air-hole diameter is decreased, the contribution of the air in the cladding decrease and hence the refractive index of the cladding approaches to Si refractive index. This leads to the decreases of core-cladding index contrast.

Based on Eq. (1), in the anomalous dispersion regime, when the value of $\beta_{2}$ is smaller, the greater value of $\Omega_{\max }$ is obtained. Therefore, with decreasing $\beta_{2}$, the frequency shift increases. This can be seen in Table (2) where the smallest value of $\beta_{2}$ corresponds to the smallest diameter of the air hole. Table (3) lists the Stokes and anti-Stokes frequencies $\left(\omega_{s}\right.$ and $\left.\omega_{a}\right)$ as well as the wavelength shifts $\Delta \lambda$, where the pump wavelength as the pump peak power is fixed at $500 \mathrm{~W}$. From Eq. (1), for smaller $\beta_{2}$, the larger $\Omega_{\max }$ is occurred, hence the larger wavelength shift of $1.22 \mu \mathrm{m}$ is obtained.

Table 3. Sidebands frequencies along with wavelength shifts for different air-hole diameters calculated at the pump wavelength of $\lambda_{P}=6.60 \mu \mathrm{m}$ in the anomalous dispersion regime.

\begin{tabular}{|c|c|c|c|}
\hline $\mathrm{d}(\mu \mathrm{m})$ & $\omega_{s}(\mathrm{THz})$ & $\omega_{a}(\mathrm{THz})$ & $\begin{array}{c}\Delta \lambda=\lambda_{s}-\lambda_{P} \\
(\mu \mathrm{m})\end{array}$ \\
\hline 4.60 & 266.37 & 304.39 & 0.47 \\
\hline 2.88 & 265.17 & 305.60 & 0.50 \\
\hline 2.00 & 264.07 & 306.69 & 0.53 \\
\hline 1.60 & 262.64 & 308.13 & 0.57 \\
\hline 1.20 & 259.16 & 311.61 & 0.67 \\
\hline 1.06 & 256.21 & 314.56 & 0.75 \\
\hline 0.94 & 250.36 & 320.40 & 0.92 \\
\hline 0.86 & 240.79 & 329.98 & 1.22 \\
\hline
\end{tabular}

Fig. 3 shows the gain coefficients as a function of the frequency detuning $(\Omega)$ simulated based on Eq. (3) for different air-hole diameters. According to Fig. 3, for $\mathrm{d}=0.86 \mu \mathrm{m}$, the largest detuning at which the gain peak of the sidebands occurs corresponds to the smallest value of $\beta_{2}$ which is already seen in Tables (2) and (3). 


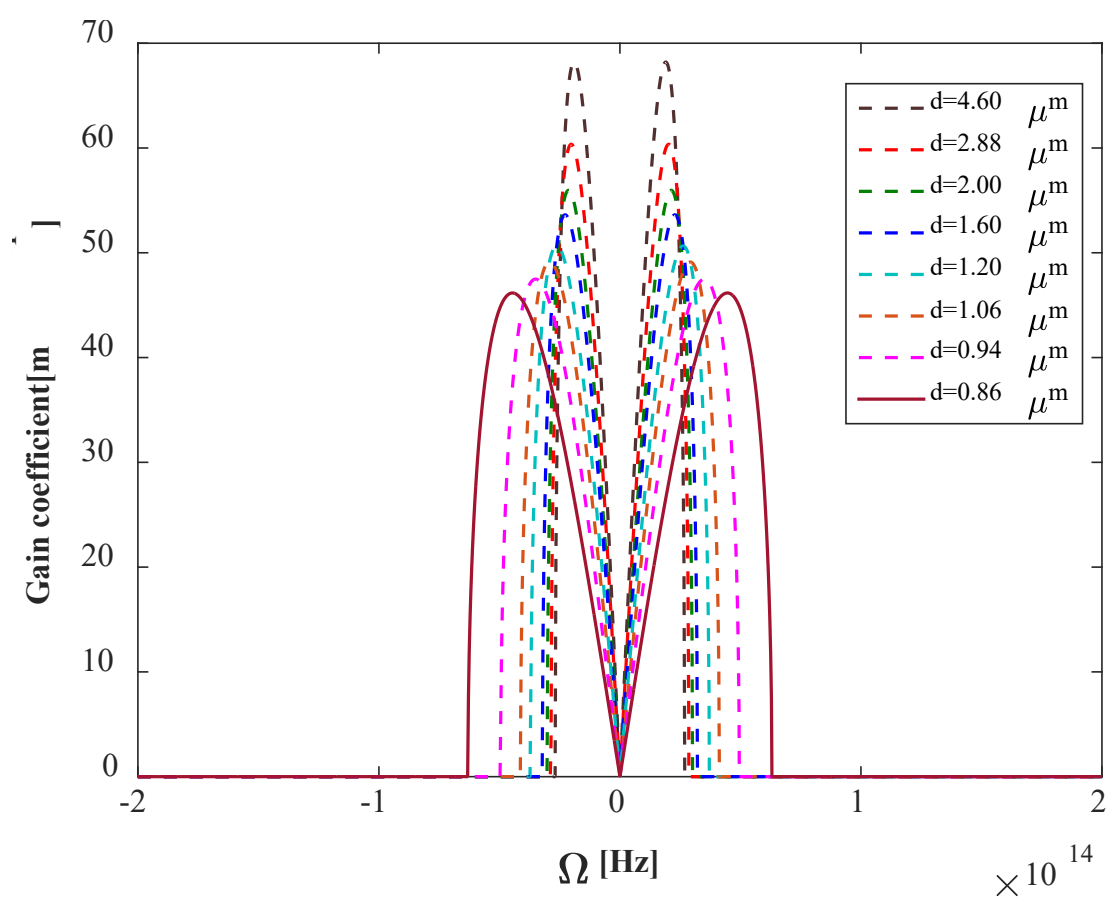

Fig 3. Gain coefficient versus the frequency detuning for various air-hole diameters in the anomalous dispersion regime. The pump wavelength is $\lambda_{\mathrm{P}}=6.60 \mu \mathrm{m}$ and the pump peak power is $\mathrm{P}_{\mathrm{p}}=500 \mathrm{~W}$.

Based on Fig. 4, the gain coefficient is increased. Additionally, increasing the pump power will shift the peak frequency detuning $\Omega_{\max }$ to larger values. This factor is due to increase of nonlinear interaction and increased phase matching between the interacting waves. It worth noting that the practical values of $\lambda_{p}$ and $P_{p}$ can be readily obtained via mid infra-red sources operating between 3 and 8 microns; for example, the second-harmonic generation of the intense $\mathrm{CO}$ laser using the $\mathrm{ZnGeP}_{2}$ crystal or coherent radiation based on difference-frequency generation from tunable optical parametric oscillators (OPOs) $[25,26]$.

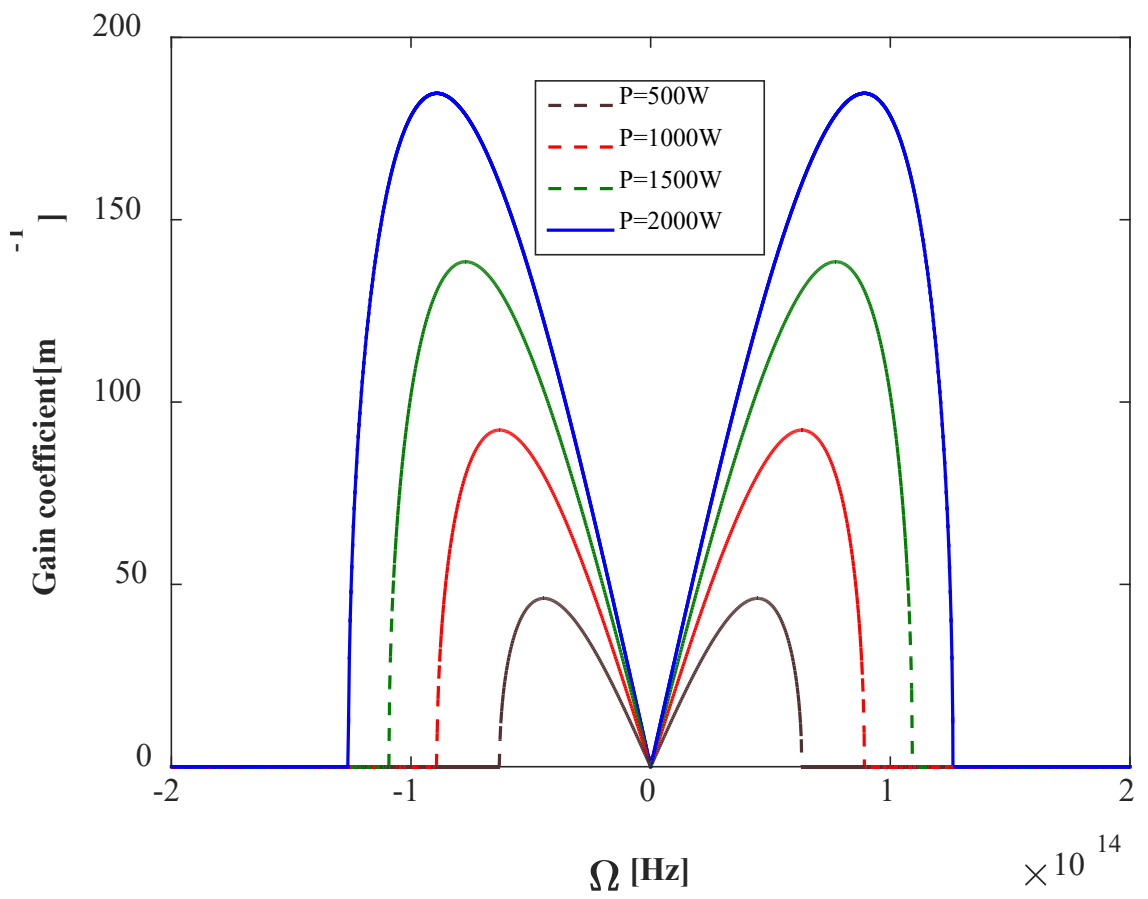


Fig 4. The gain coefficient versus the frequency detuning for different pump peak powers in the anomalous dispersion regime. The air-hole diameter is fixed at $\mathrm{d}=0.86 \mu \mathrm{m}$.

As discussed above, in the PC-based rib silicon waveguide, it is possible to tune the converted wavelength by changing the waveguide geometry. In this case, the largest sideband peak was obtained for $\mathrm{d}=0.86 \mu \mathrm{m}$ at which $\beta_{2}$ has the smallest value, and correspondingly the largest wavelength shift of $\Delta \lambda$ $=1.22 \mu \mathrm{m}$ is observed. Also, with increasing pump peak power for this fixed air-hole diameter, the frequency shift is increased. Therefore, it is possible to control the location of the converted wavelengths by changing the air-hole diameter as well as the input pump peak power. However, as it is seen from the simulation results, the largest wavelength shift occurred in the anomalous dispersion regime cannot reach the terahertz region; therefore, one should examine the wavelength conversion in the normal dispersion regime.

\section{4-2 Tunable Wavelength Conversion via Geometrical Change in Normal Dispersion Regime}

In this section, we change the pump wavelength and repeat simulations for $\lambda_{\mathrm{P}}=5.60 \mu \mathrm{m}$. This is because at this wavelength, for all air-hole diameters according to Table (1), the normal dispersion regime is fulfilled, i.e., $\lambda_{\mathrm{p}}<\mathrm{ZDW}$. In this regime, $\beta_{2}$ is positive and $\beta_{4}$ is negative [24]. The dispersion and nonlinear parameters of the PC-based rib silicon waveguide are listed in Table (4); while the wavelengths shift and Stokes and anti-stokes frequencies are given in Table (5).

Table 4. Dispersion and nonlinear parameters of the PC-based rib silicon waveguide for different diameters of air holes at the pump wavelength of $\lambda_{\mathrm{P}}=5.60 \mu \mathrm{m}$ in normal dispersion regime.

\begin{tabular}{|c|c|c|r|c|}
\hline $\mathrm{d}(\mu \mathrm{m})$ & $\begin{array}{c}\beta_{2} \\
\left(\mathrm{ss}^{2} / \mathrm{m}\right)\end{array}$ & \multicolumn{1}{c|}{$\begin{array}{c}\beta_{4} \\
\left(\mathrm{ps}^{4} / \mathrm{m}\right)\end{array}$} & $\begin{array}{c}A_{\text {eff }} \\
\left((\mu \mathrm{m})^{2}\right)\end{array}$ & $\begin{array}{c}\gamma \\
(1 / W m)\end{array}$ \\
\hline 4.60 & $8.64 \times 10^{-3}$ & $-2.48 \times 10^{-5}$ & 68.94 & $8.13 \times 10^{-2}$ \\
\hline 2.88 & $3.45 \times 10^{-2}$ & $-2.24 \times 10^{-5}$ & 77.84 & $7.20 \times 10^{-2}$ \\
\hline 2.00 & $4.89 \times 10^{-2}$ & $-2.06 \times 10^{-5}$ & 83.86 & $6.69 \times 10^{-2}$ \\
\hline 1.60 & $5.96 \times 10^{-2}$ & $-1.90 \times 10^{-5}$ & 87.37 & $6.42 \times 10^{-2}$ \\
\hline 1.20 & $7.50 \times 10^{-2}$ & $-1.61 \times 10^{-5}$ & 92.06 & $6.09 \times 10^{-2}$ \\
\hline 1.06 & $8.28 \times 10^{-2}$ & $-1.45 \times 10^{-5}$ & 94.60 & $5.93 \times 10^{-2}$ \\
\hline 0.94 & $9.21 \times 10^{-2}$ & $-1.26 \times 10^{-5}$ & 97.32 & $5.76 \times 10^{-2}$ \\
\hline 0.86 & $9.96 \times 10^{-2}$ & $-1.09 \times 10^{-5}$ & 99.58 & $5.63 \times 10^{-2}$ \\
\hline
\end{tabular}

As before, by decreasing the air-hole diameter, the core-cladding index contrast is decreased and so Aeff is increased and simultaneously $\gamma$ decreases. According to Eq. (2), in the normal dispersion regime, the peak frequency detuning depends on both the second- and the fourth-order dispersion coefficients so that for the largest $\beta_{2}$ and the smallest $\beta_{4}, \Omega_{\max }$ becomes maximum. As is seen in Table (4), the largest $\beta_{2}$ and 
simultaneously the smallest $\beta 4$ correspond to the smallest value of air-hole diameter with $\mathrm{d}=0.86 \mu \mathrm{m}$. This leads to the maximum wavelength shift of $\Delta \lambda=320.57 \mu \mathrm{m}$ which is given in Table (5).

Table 5. Stokes and anti-Stokes frequencies as well as the wavelength shifts for different air-hole diameters for the pump wavelength of $\lambda_{\mathrm{P}}=5.60 \mu \mathrm{m}$ in the normal dispersion regime.

\begin{tabular}{|c|c|c|c|}
\hline $\mathrm{d}(\mu \mathrm{m})$ & $(\mathrm{THz}) \omega_{s}$ & $\omega_{a}(\mathrm{THz})$ & $\Delta \lambda(\mu \mathrm{m})$ \\
\hline 4.60 & 243.12 & 429.60 & 2.15 \\
\hline 2.88 & 193.57 & 479.15 & 4.13 \\
\hline 2.00 & 163.87 & 508.85 & 5.90 \\
\hline 1.60 & 139.55 & 533.17 & 7.90 \\
\hline 1.20 & 98.47 & 574.25 & 13.54 \\
\hline 1.06 & 73.37 & 599.35 & 20.09 \\
\hline 0.94 & 38.99 & 633.73 & 42.74 \\
\hline 0.86 & 5.78 & 666.94 & 320.57 \\
\hline
\end{tabular}

As a result, it is desired to work in the normal dispersion regime to access new wavelengths in the $\mathrm{THz}$ region.

\section{4-3 Tunable Wavelength Conversion via Optofluidic Infiltration}

As discussed above, one can tailor the waveguide dispersion curve by changing the geometrical parameters and hence tune the generated wavelength range. However, by the infiltration of optical fluids with different refractive indices as the post-fabrication method, one can easily engineer linear and nonlinear characteristics of the waveguide to access the desired wavelength range. From the practical point of view, it is a more appropriate and optimal tool that enables us to achieve significant results via small manipulations. The variation of waveguide geometry is not an optimal method for reaching different wavelength regions, because one cannot practically use a waveguide with different air-hole diameters. Therefore, we should think of changing the dispersion properties of the waveguide in a different way, such as the infiltration of optical fluids to access different wavelength regions while keeping the waveguide geometry unchanged.

In this section, we set the waveguide dimensions at fixed parameters of $\Lambda=4.8 \mu \mathrm{m}$ and $\mathrm{d}=0.86 \mu \mathrm{m}$, to ensure the largest wavelength shift as discussed in the previous section; and then infiltrate different optical fluids with various refractive indices in the PC air holes. To this end, we infiltrate water or other optical fluids into the PC air holes and then simulate $\beta_{2}$ and $\beta_{4}$ curves and compare them with the case of empty air holes (without infiltration). This is shown in Fig. 5 (a) whereby increasing the refractive index of the holes, the dispersion curve is changed and ZDW is shifted toward longer wavelengths. For example, for the empty air hole, the ZDW is about $6.40 \mu \mathrm{m}$ while for the optical fluid of $C_{8} H_{18}$ this value is shifted to $6.56 \mu \mathrm{m}$.

As shown in Figs. 5 (b) and (c), $\beta_{2}$ and $\beta_{4}$ are plotted for both cases when the waveguide is uninfiltrated or infiltrated with various optical fluids. Evidently, by changing the refractive index of the fluid infiltrated into the PC air holes, the second- and fourth-order dispersion coefficients are changed and hence the wavelength shift is tuned according to Eq. (2). 

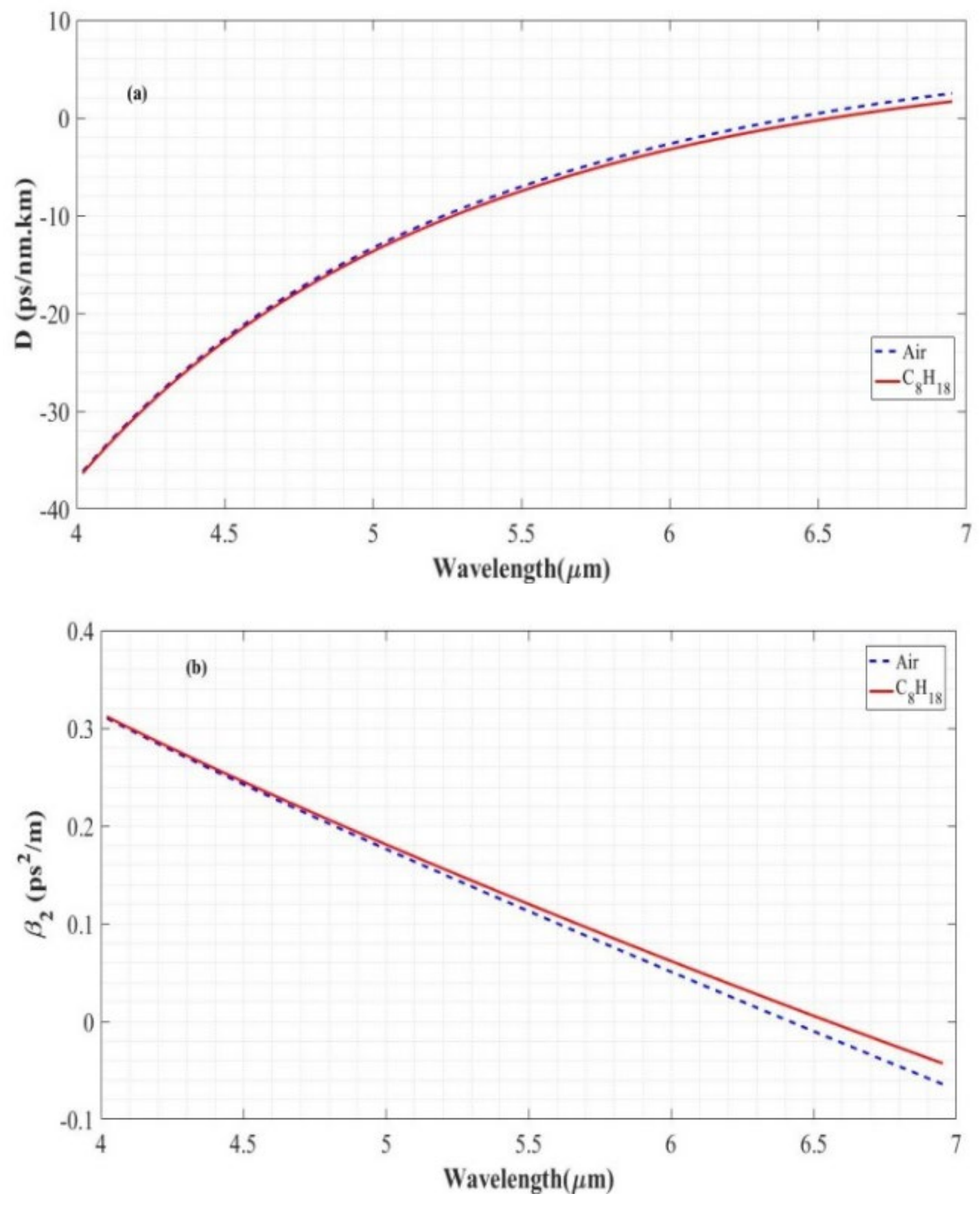


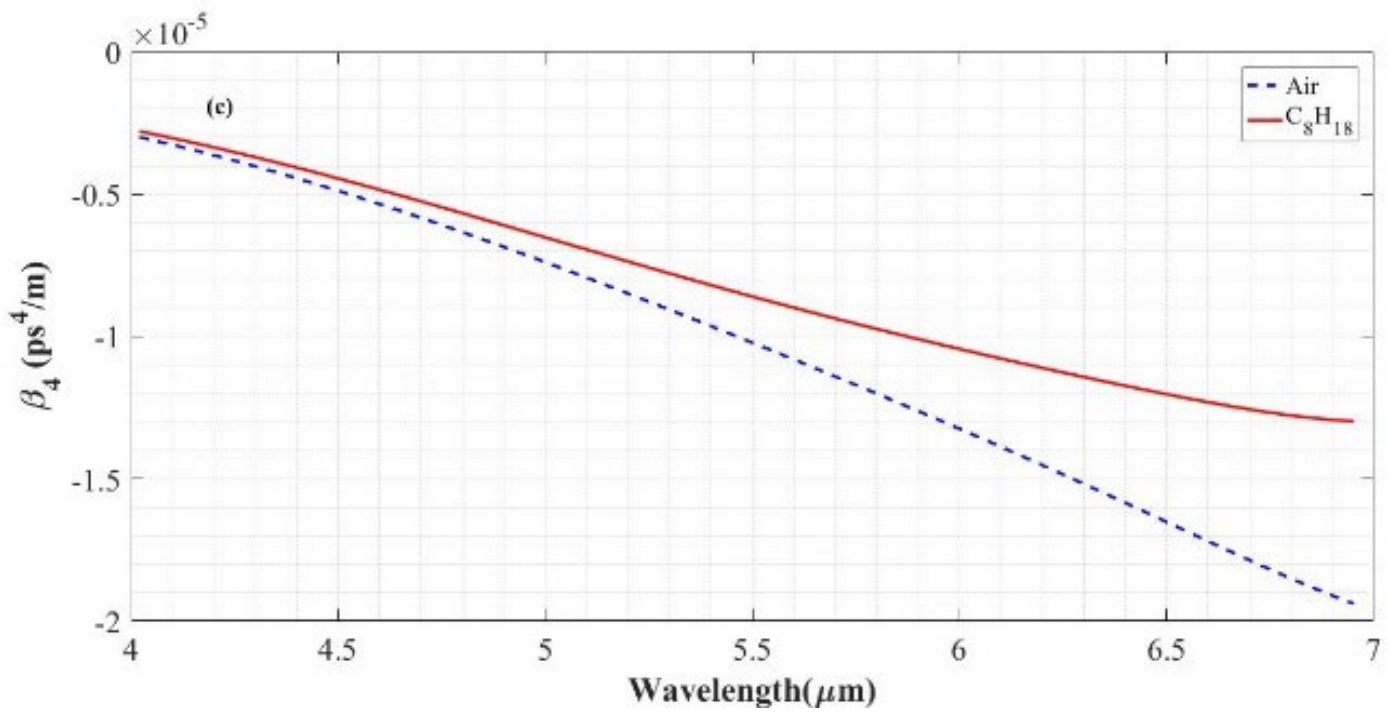

Fig 5. Dispersion curves (a); GVD parameters (b); and fourth-order dispersion coefficients (c) for empty air hole (blue line) and optofluidic-infiltrated air hole (red line).

Based on the obtained results in Sections 4-1 and 4-2, we obtain a larger wavelength shift for the normal dispersion regime. Therefore, in this section, simulations are only performed in the normal dispersion regime. Here, three different optical fluids including water, $\mathrm{C}_{5} \mathrm{H}_{12}$ and $\mathrm{C}_{8} \mathrm{H}_{18}$ are infiltrated into the PC air holes. The refractive indices of these fluids are as $n_{\text {water }}=1.33400$ [27], $n_{C_{5} H_{12}}=1.35377$ [28] and $n_{C_{8} H_{18}}=1.39125$ [28]. The pump wavelength is set at $\lambda_{\mathrm{P}}=5.9 \mu \mathrm{m}$, where for all cases the normal dispersion regime, $\lambda_{\mathrm{P}}<\mathrm{ZDW}$, takes place and therefore the second-order dispersion coefficient is positive and simultaneously the fourth-order dispersion coefficient is negative.

The linear and nonlinear parameters for all cases of un-infiltrated and infiltrated $\mathrm{PC}$ holes are listed in Table (6) and corresponding wavelengths shifts, Stokes and anti-stokes frequencies are given in Table (7).

Table 6. Linear and nonlinear parameters of the PC-based rib silicon waveguide infiltrated by optical fluids with different refractive indices. The pump wavelength is set at $\lambda_{\mathrm{P}}=5.9 \mu \mathrm{m}$ in the normal dispersion regime.

\begin{tabular}{|c|c|c|c|c|}
\hline Optical fluid & $\begin{array}{c}\beta_{2} \\
\left(p s^{2} / m\right)\end{array}$ & $\begin{array}{c}\beta_{4} \\
\left(p s^{4} / m\right)\end{array}$ & $\begin{array}{c}A_{\text {eff }} \\
\left((\mu m)^{2}\right)\end{array}$ & $\begin{array}{c}\gamma \\
(1 / W . m)\end{array}$ \\
\hline Air & $6.45 \times 10^{-2}$ & $-1.26 \times 10^{-5}$ & 100.77 & $5.29 \times 10^{-2}$ \\
\hline Water & $7.27 \times 10^{-2}$ & $-1.05 \times 10^{-5}$ & 102.22 & $5.22 \times 10^{-2}$ \\
\hline$C_{5} H_{12}$ & $7.33 \times 10^{-2}$ & $-1.04 \times 10^{-5}$ & 102.32 & $5.21 \times 10^{-2}$ \\
\hline$C_{8} H_{18}$ & $7.45 \times 10^{-2}$ & $-1.01 \times 10^{-5}$ & 102.53 & $5.20 \times 10^{-2}$ \\
\hline
\end{tabular}

By increasing the refractive index of the air holes through the injection of optical fluid, the index contrast between the core and cladding decreases and so Aeff increases and $\gamma$ decreases. As mentioned above, according to Eq. (2), in the normal dispersion regime, the peak frequency shift depends on both secondand fourth-order dispersion coefficients so that when $\beta_{2}$ is larger and $\beta_{4}$ is smaller, $\Omega_{\max }$ is larger as well. As seen in Table (6), the largest value of $\beta_{2}$ and the smallest value of $\beta_{4}$ correspond to the case of $C_{8} H_{18}$ where according to Table (7), this leads to the largest wavelength shift of $\Delta \lambda=101.58 \mu \mathrm{m}$. This largest 
wavelength shift corresponds to the maximum converted wavelength of $\lambda=107.48 \mu \mathrm{m}$ which lies in the THz region.

Table 7. Stokes and anti-Stokes frequencies as well as the wavelength shifts of the PC-based rib silicon waveguide infiltrated by optical fluids with different refractive indices. The pump wavelength is set at $\lambda_{P}=5.9 \mu \mathrm{m}$ in the normal dispersion regime.

\begin{tabular}{|c|l|c|c|}
\hline Optical fluid & $\omega_{s}(\mathrm{THz})$ & $\omega_{a}(\mathrm{THz})$ & $\Delta \lambda(\mu \mathrm{m})$ \\
\hline Air & 65.71 & 574.45 & 22.80 \\
\hline Water & 26.75 & 613.42 & 64.59 \\
\hline$C_{5} H_{12}$ & 24.54 & 615.62 & 70.92 \\
\hline$C_{8} H_{18}$ & 17.54 & 622.62 & 101.58 \\
\hline
\end{tabular}

Fig. 6 shows a gain coefficient versus the frequency detuning simulated based on Eq. (4) for both cases: $\mathrm{C}_{8} \mathrm{H}_{18}$ optofluidic infiltration and empty air holes. As it is obvious, by infiltration of the optical fluid, the sideband peak frequency detuning is largest compared with the empty air hole. Therefore, the designed PC-based rib silicon waveguide can generate different wavelength shifts by changing the refractive index of the air holes via optofluidic infiltration. This provides an efficient method to access the $\mathrm{THz}$ region without changing the geometrical parameters.

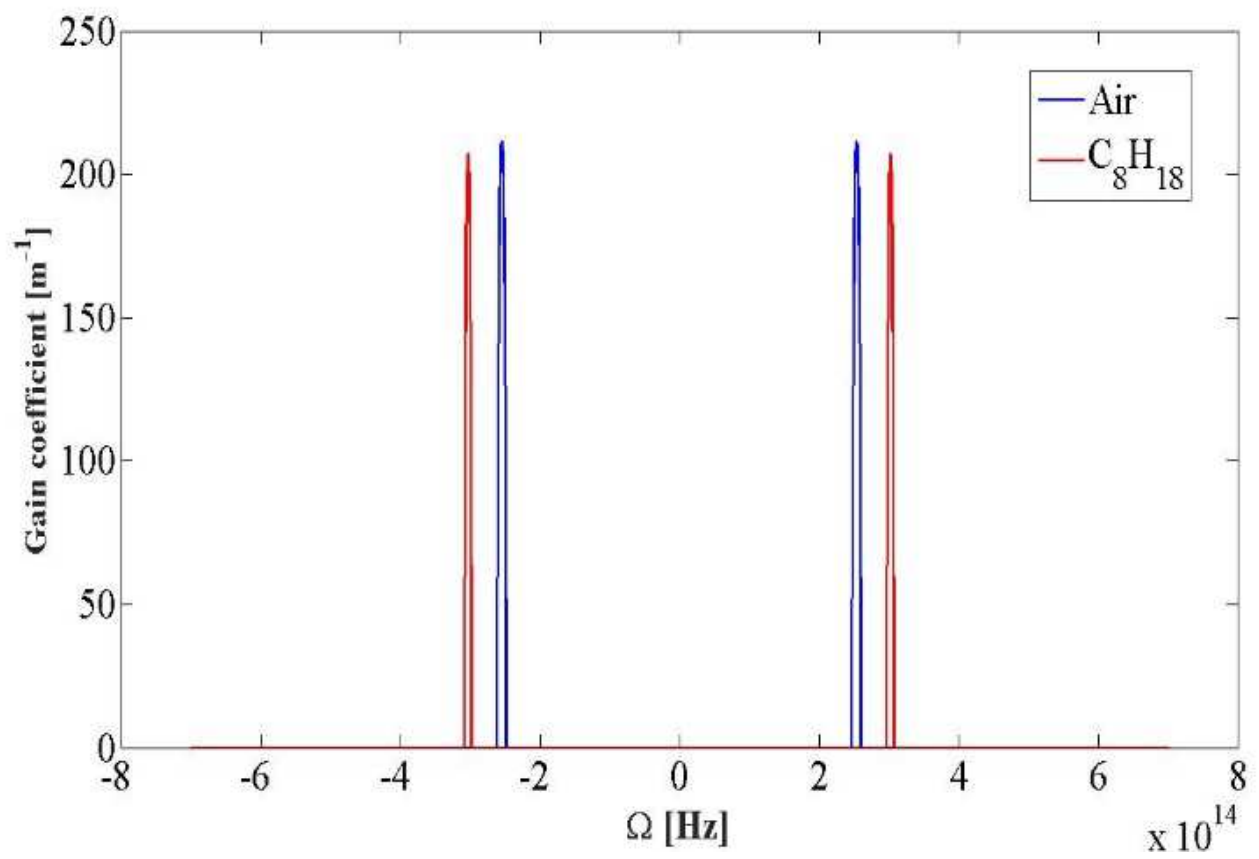

Fig 6. Gain coefficient versus the frequency detuning for empty air hole (blue line) and optofluidic infiltrated air hole (red line). The pump wavelength is set at $\lambda_{\mathrm{P}}=5.9 \mu \mathrm{m}$ in the normal dispersion regime with the pump peak power of $\mathrm{P}_{\mathrm{p}}=2 \mathrm{~kW}$.

\section{Conclusion}

In this paper, a rib silicon waveguide based on the photonic crystal (PC) idea was designed for the first time and tunable wavelength conversion for generation of $\mathrm{THz}$ radiation was simulated. As a result, the converted frequencies/wavelengths can easily lie in the THz region. When the pump wavelength is set at 
$\lambda_{\mathrm{p}}=5.60 \mu \mathrm{m}$ in the normal dispersion regime and for the fixed lattice pitch of $\Lambda=4.80 \mu \mathrm{m}$, as the air-hole diameter is changed from $\mathrm{d}=4.60 \mu \mathrm{m}$ to $\mathrm{d}=0.86 \mu \mathrm{m}$, the converted wavelength is tuned from $\lambda=2.15 \mu \mathrm{m}$ to $\lambda=326.17 \mu \mathrm{m}$, respectively. This is while for the pump wavelength of $\lambda_{\mathrm{p}}=5.9 \mu \mathrm{m}$ and the fixed lattice pitch of $\Lambda=4.80 \mu \mathrm{m}$ and air-hole diameter of $\mathrm{d}=0.86 \mu \mathrm{m}$, by the infiltration of optical fluids with different refractive indices in the PC air holes, the converted wavelength is tuned over the $\mathrm{THz}$ range from $70.5 \mu \mathrm{m}$ to $107.5 \mu \mathrm{m}$.

\section{Funding}

No funding was received for this work.

\section{Author contributions}

All authors contributed to the study conception and design. Material preparation, simulation results and analysis were performed by H. Pakarzadeh and S. Hosseinabadi. The first draft of the manuscript was written by S. Hosseinabadi and all authors commented on previous versions of the manuscript. All authors read and approved the final manuscript.

\section{Availability of data and material}

Data are available on request from the authors.

\section{Compliance with ethical standards}

Compliance with ethical standards is followed by authors.

\section{- Disclosure of potential conflicts of interest}

The authors declare that they have no potential conflicts of interests.

\section{- Research involving Human Participants and/or Animals}

Not applicable.

\section{- Informed consent}

Not applicable.

\section{Consent to participate}

Not applicable.

\section{Consent for Publication}

Not applicable.

\section{Acknowledgments}

Not applicable.

\section{References}

[1] Zhang X. C., Xu J., 2010, Springer Science+ Business Media LLC. 
[2] Pawar, A. Y., Sonawane, D. D., Erande, K. B. and Derle, D. V., 2013, Drug invention today, 5(2), 157-163.

[3] Lewis R. A., 2014, Journal of Physics D, 47(37), 374001.

[4] Burford, N.M. and El-Shenawee, M.O., 2017, Optical Engineering, 56(1), 010901.

[5] Yang, X., Brunetti, E. and Jaroszynski, D.A. 2018,_New Journal of Physics, 20(4), 043046.

[6] Evain, C., Szwaj, C., Roussel, E., Rodriguez, J., Le Parquier, M., Tordeux, M.A., Ribeiro, F., Labat, M., Hubert, N., Brubach, J.B. and Roy, P., 2019, Nature. Physics, 15(7), 635-639.

[7] Franckié M., and Faist J., 2020, Physical Review Applied 13(3), 034025.

[8] Ravi K., Huang W.R., Carbajo S., Nanni E.A., Schimpf D.N., Ippen E.P. and Kärtner F.X., 2015, Optics Express, 23(4), 5253-5276.

[9] Piyathilaka H.P., Sooriyagoda R., Dewasurendra V., Johnson M.B., Zawilski K.T., Schunemann P.G. and Bristow A.D., 2019, Optics Express, 27(12), 16958-16965.

[10] Wang Z., Liu L., Huang N., Sun Q. and Wen J., 2012, Optics Express., 20(8), 8920-8928.

[11] Soref R. A., Emelett S. J. and Buchwald W. R., 2006, Journal of Optics A: Pure and Applied Optics, 8(10), 840-848.

[12] Yin L., Lin Q., and Agrawal G. P., 2007, Optics Letters 32(4), $391-393$.

[13] Agrawal G. P., 2013, $5^{\text {th }}$ Edition, Academic Press, San Diago.

[14] Psaltis D., Quake S. R. and Yang C., 2006, Nature, 442(7101), 381-386.

[15] Ebnali-Heidari M., Dehghan F., Saghaei H., Koohi-Kamali F. and Moravvej-Farshi M. K., 2012, Journal of Modern Optics, 59(16), 1384-1390.

[16] Pakarzadeh H., Derakhshan R. and Hosseinabadi S., 2019, Journal of Nonlinear Optical Physics \& Materials, 28(1), 1950002.

[17] Casas Bedoya A., Domachuk P., Ting J., Grillet C., Monat C., Tomljenovic-Hanic S., Lee M. W., McPhedran R. C., and Eggleton B. J., 2010, In Conference on Lasers and Electro-Optics, CWM4.

[18] Tatian, B., 1984, Applied Optics, 23(24), 4477-4485.

[19] Crisp J. and Elliott B., 2005, $3^{\text {rd }}$ Edition, Elsevier.

[20] Zhu Z. and Brown T. G., 2002, Optics Express., 10(17), 853-864. 
[21] Pakarzadeh H., Rezaei S. M., Namroodi L., 2019, Optics Communications, 433, 81-88.

[22] K. Saitoh and M. Koshiba, 2005, Journal of Lightwave Technology, 23(11), 3580-3590.

[23] Pakarzadeh, H. and Rezaei, S.M., 2016, Journal of Modern Optics, 63(2), 151-158.

[24] Pakarzadeh H., Hosseinabadi S. and Amiri I.S., 2019, Proc. SPIE 11205, Seventh International Conference on Optical and Photonic Engineering (icOPEN 2019), 1120504.

[25] Ionin, A. A., Kinyaevskii, I.O., Klimachev, Y.M., Kozlov, A.Y. and Kotkov, A.A., 2017, Physics of Atomic Nuclei, 80(11), 1635-1641.

[26] Ebrahim-Zadeh, M. and Sorokina I. T., 2008, Springer.

[27] Hale G. M. and Querry M. R., 1973, Applied Optics, 12, 555-563.

[28] Anderson M. R., 2000, Master Thesis, University of Missouri-Rolla, USA. 


\section{Figures}

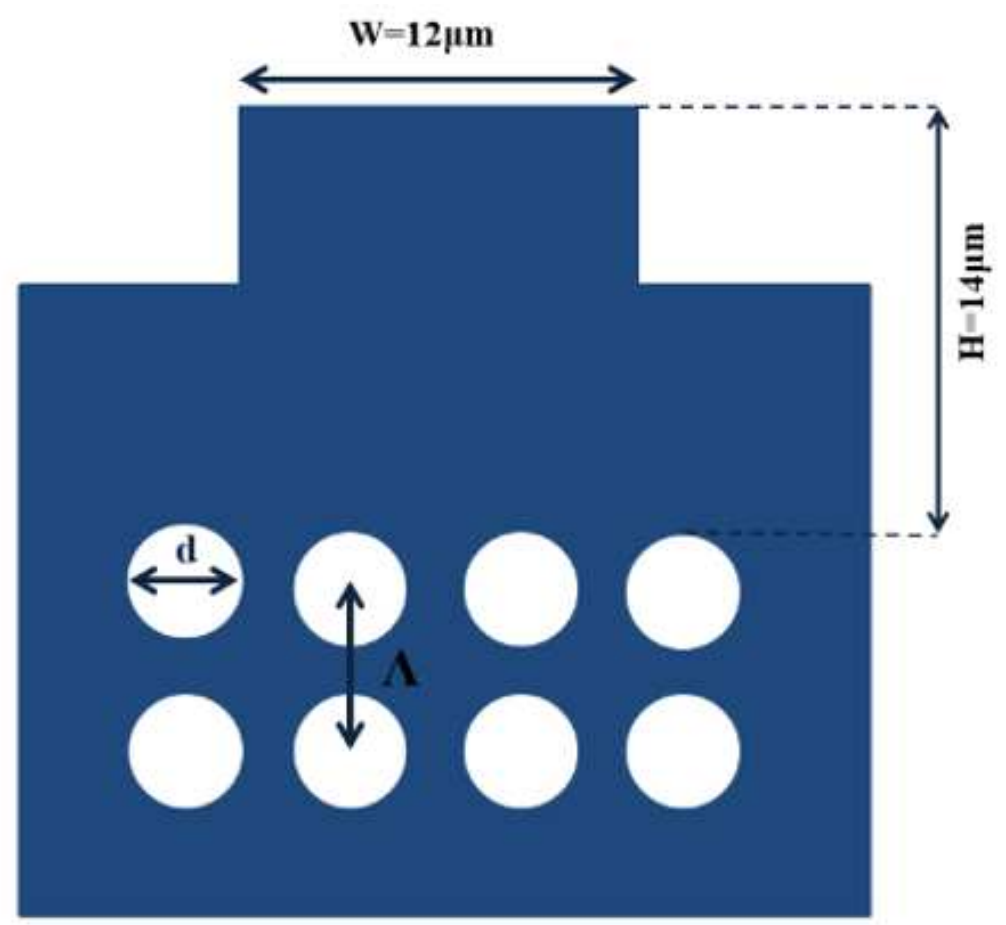

(a)

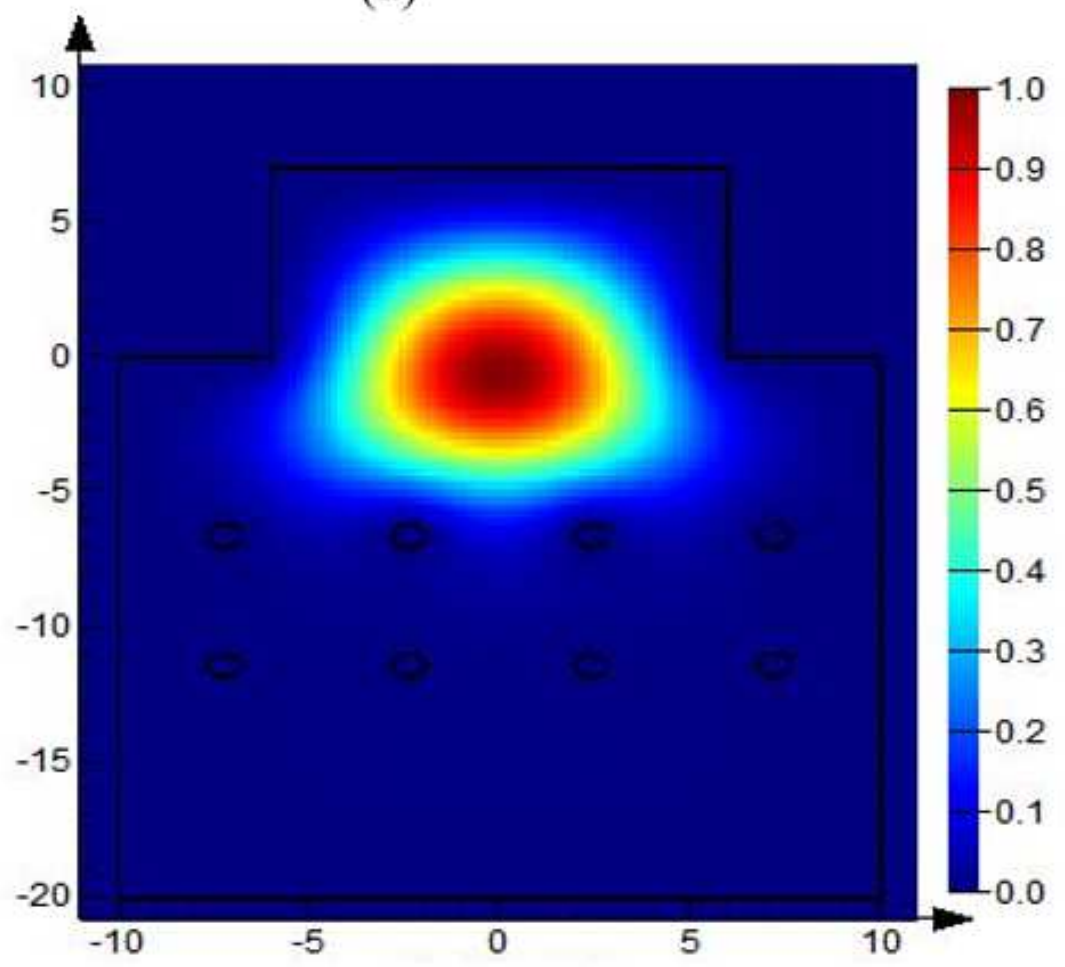

(b)

Figure 1

(a) Cross-sectional view of the PC-based rib silicon waveguide; (b) distribution of the fundamental TM mode confined in the waveguide core. The air-hole diameter $\mathrm{d}$ can be changed for geometrical change of waveguide. 


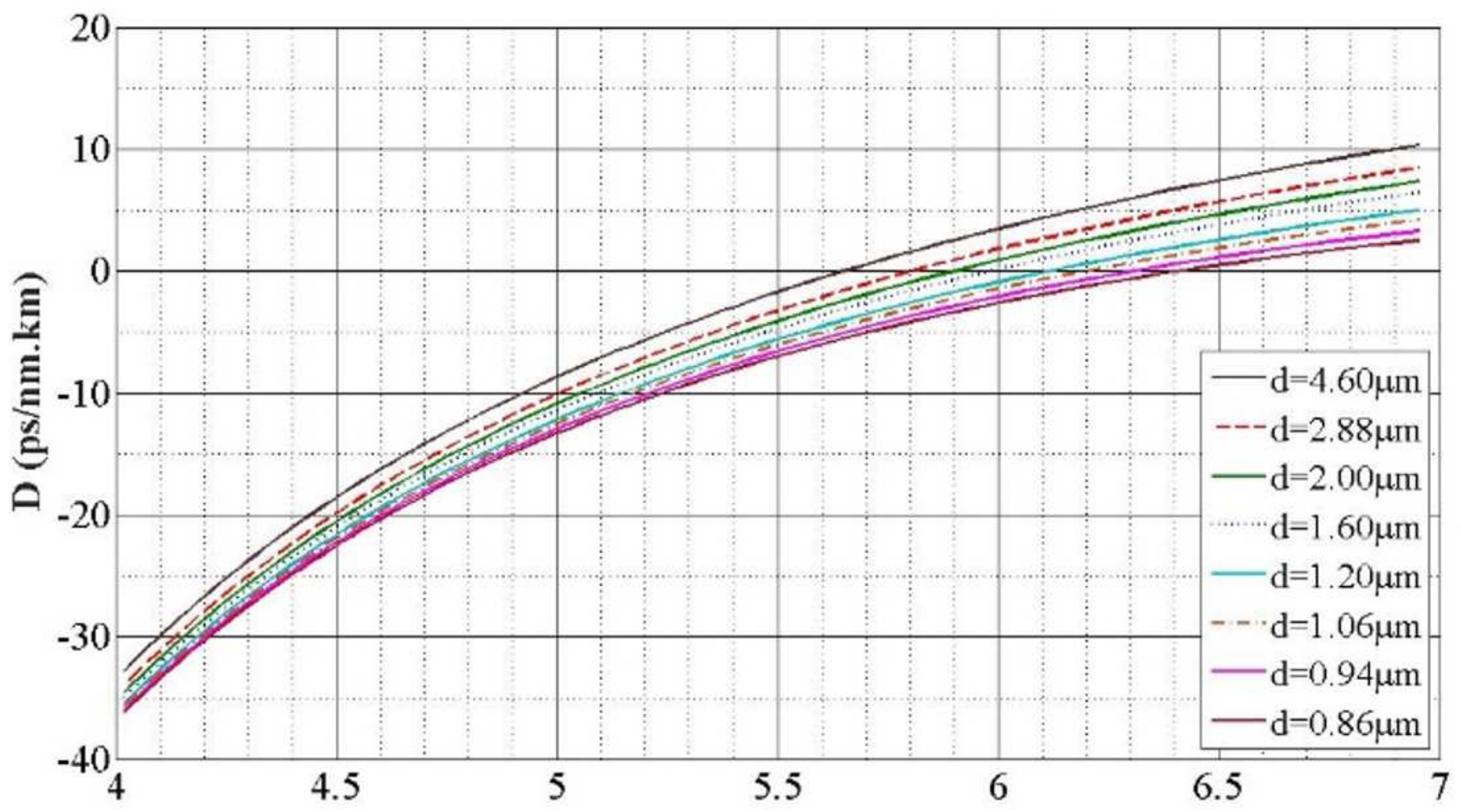

Figure 2

Dispersion curves D versus wavelength for different air-hole diameters $d$. 


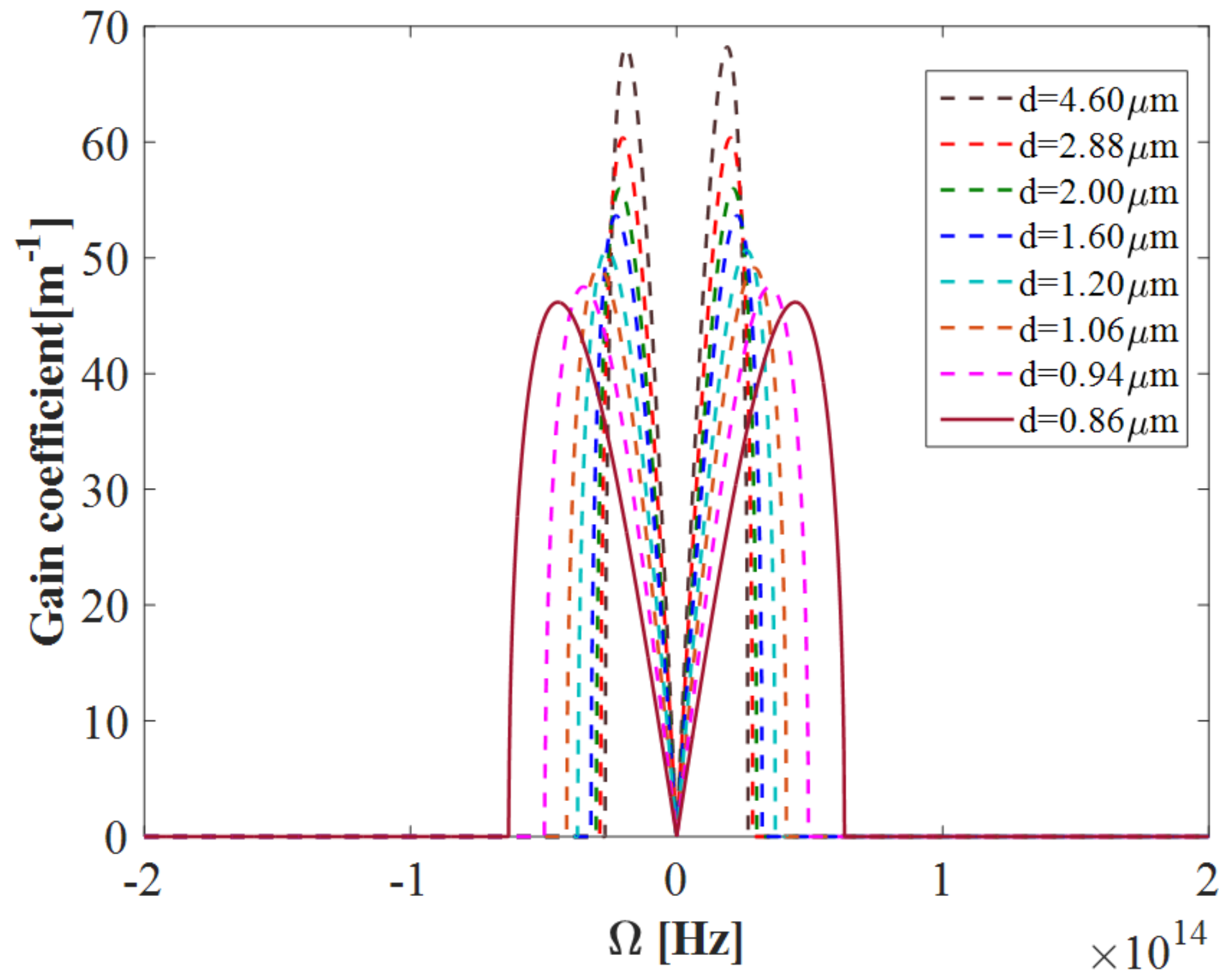

Figure 3

Gain coefficient versus the frequency detuning for various air-hole diameters in the anomalous dispersion regime. The pump wavelength is $\lambda \mathrm{P}=6.60 \mu \mathrm{m}$ and the pump peak power is $\mathrm{Pp}=500 \mathrm{~W}$. 


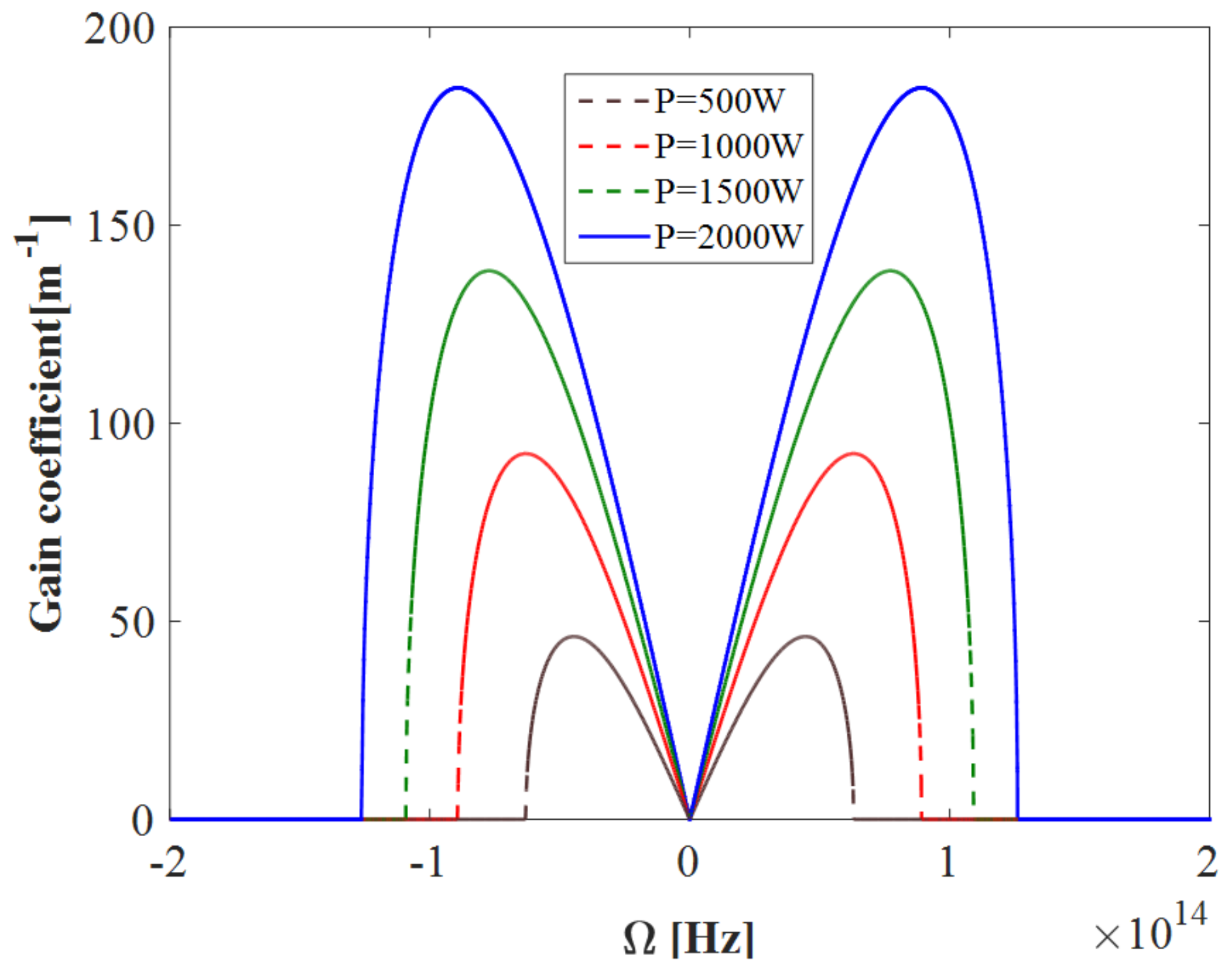

Figure 4

The gain coefficient versus the frequency detuning for different pump peak powers in the anomalous dispersion regime. The air-hole diameter is fixed at $d=0.86 \mu \mathrm{m}$. 

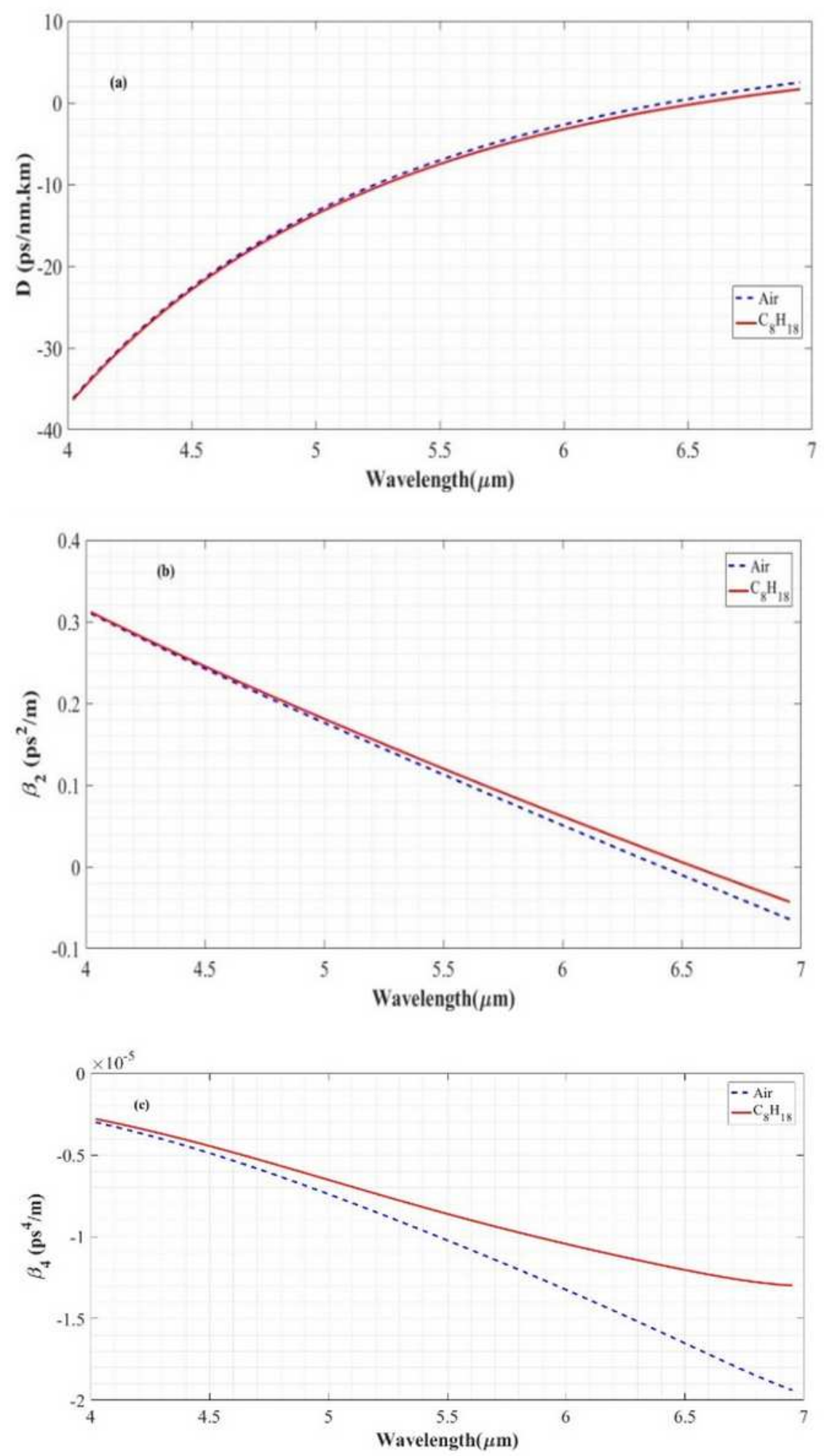

Figure 5

Dispersion curves (a); GVD parameters (b); and fourth-order dispersion coefficients (c) for empty air hole (blue line) and optofluidic-infiltrated air hole (red line). 


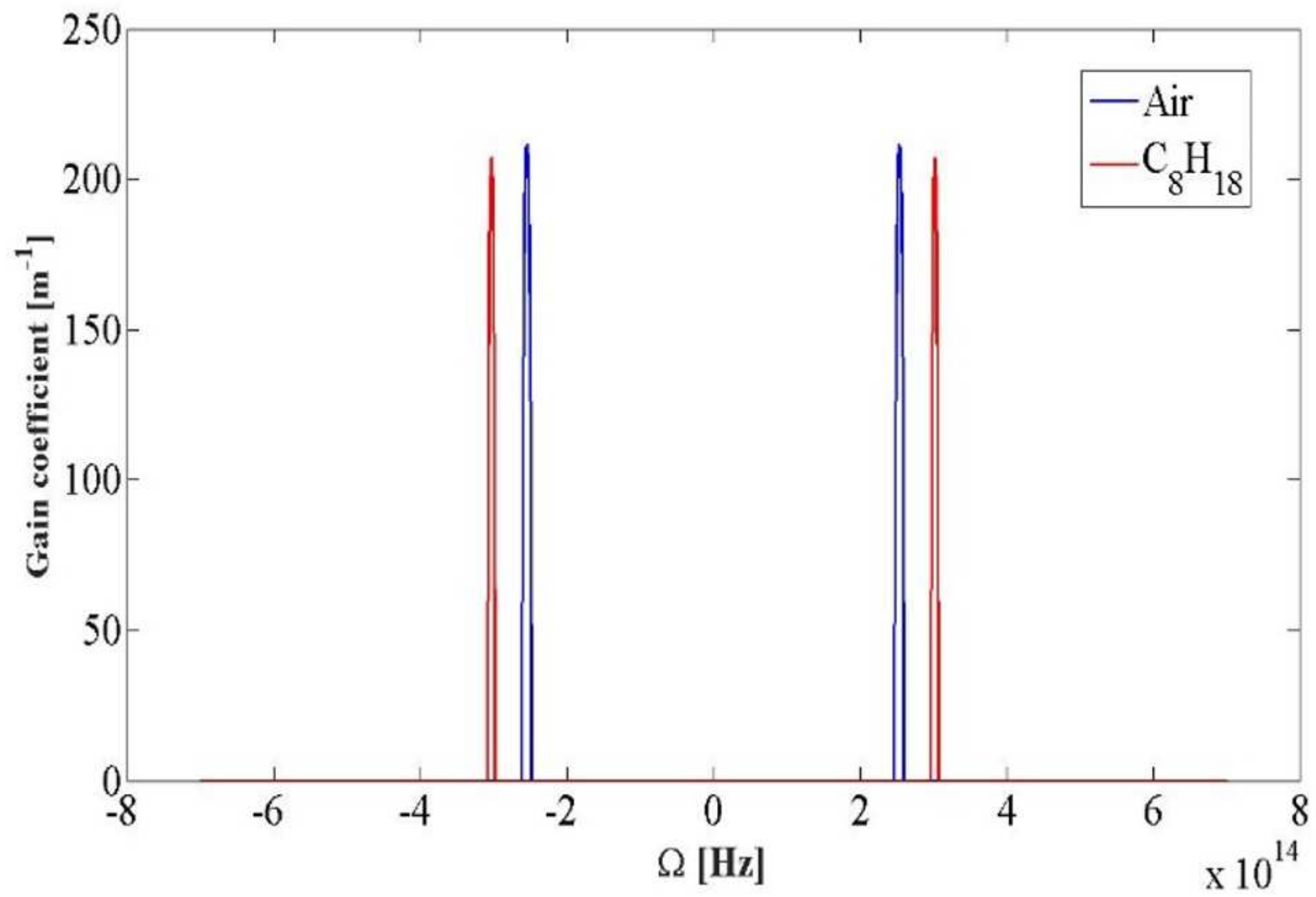

Figure 6

Gain coefficient versus the frequency detuning for empty air hole (blue line) and optofluidic infiltrated air hole (red line). The pump wavelength is set at $\lambda P=5.9 \mu \mathrm{m}$ in the normal dispersion regime with the pump peak power of $\mathrm{Pp}=2 \mathrm{~kW}$. 